\title{
Implementación de TIC'S para la gestión de Visitas de divulgación de la científica
}

\section{Implementation of TIC'S for the management of Science Outreach Visits}

\author{
MAGDALENO-ZAVALA, Juan Antonio $\dagger^{*}$, DURAN-BELMAN, Israel y VARGAS-ESPINOZA, \\ Elizabeth Gabriela
}

Instituto Tecnológico Superior de Irapuato, Carretera Irapuato-Silao Km 12.5, Colonia el Copal

ID $1^{\mathrm{er}}$ Autor: Juan Antonio, Magdaleno-Zavala / ORC ID: 0000-0001-9762-0086, Researcher ID Thomson: S-5543-2018, CVU CONACYT ID: 691690

ID $1^{\text {er }}$ Coautor: Israel, Duran-Belman / ORC ID: 0000-0002-1394-0486, Researcher ID Thomson: V-7806-2019, CVU CONACYT ID: 691483

ID $2^{\text {do }}$ Coautor: Elizabeth Gabriela, Vargas-Espinoza / ORC ID: 0000-0003-4509-2052, Researcher ID Thomson: S-47912018, CVU CONACYT ID: 946754

DOI: $10.35429 /$ JIT.2019.18.6.17.22

Recibido: 10 de Enero, 2019; Aceptado 30 de Marzo, 2019

\section{Resumen}

El Instituto Tecnológico Superior de Irapuato cuenta con el programa "Laboratorio Itinerante", proyecto que realiza diversas actividades de divulgación científica que son ofertadas a instituciones educativas del estado de Guanajuato; Sin embargo la mala comunicación entre Responsables llevó a diversos problemas que impidieron otorgar los servicios de este laboratorio itinerante; en el año 2017 se realizaron un total de 32 solicitudes por parte de escuelas primarias y secundarias, de las cuales solo se atendieron 12, debido a la falta de organización y el tiempo que tomaba la realización del trámite. Con el fin de mitigar estos retrasos y aumentar el número de visitas, se decidió hacer uso de las TI diseñando un sistema web a través del cual las instituciones educativas puedan solicitar visitas. La metodología seguida para el desarrollo del software está basada en el ciclo de vida en cascada; se culminó con un sistema web que administra las solicitudes de visitas así como las actividades lúdicas existentes además de proporcionar reportes de atención y cantidad de personas beneficiadas, dando como resultado un incremento en las solicitudes recibidas en un $30 \%$ y la atención a éstas con un incremento del $40 \%$ comparadas con el año anterior.

\begin{abstract}
Astract
The Irapuato Higher Technological Institute has the "Itinerant Laboratory" program, a project that carries out various scientific dissemination activities that are offered to educational institutions in the state of Guanajuato; However, poor communication between Responsible led to various problems that prevented the provision of the services of this itinerant laboratory; In 2017, a total of 32 applications were made by primary and secondary schools, of which only 12 were met, due to the lack of organization and the time it took to complete the process. In order to mitigate these delays and increase the number of visits, it was decided to use IT by designing a web system through which educational institutions can request visits. The methodology followed for software development is based on the cascade life cycle; It culminated with a web system that manages the requests for visits as well as the existing recreational activities in addition to providing reports of attention and number of people benefited, resulting in an increase in requests received by $30 \%$ and attention to them with a $40 \%$ increase compared to the previous year.
\end{abstract}

Citación: MAGDALENO-ZAVALA, Juan Antonio, DURAN-BELMAN, Israel y VARGAS-ESPINOZA, Elizabeth Gabriela. Implementación de TIC'S para la gestión de Visitas de divulgación de la científica. Revista de Tecnologías de la Información. 2019. 6-18: 17-22

\footnotetext{
*Correspondencia del autor (correo electrónico: anmagdaleno@itesi.edu.mx)

$\dagger$ Investigador contribuyendo como primer autor.
} 


\section{Introducción}

La informática ha contribuido de manera esencial a la automatización de procesos, cuyo principal objetivo es mejorar la eficiencia de la empresa en relación con las expectativas de los clientes. En un proceso de automatización, el computador es la herramienta fundamental, y deberá ser potenciado con la plataforma adecuada, todo ello en consonancia con la visión y la estrategia corporativa a nivel de informática. La automatización consiste en tener a mano una información en tiempo real que sea accesible a todo el personal involucrado en la operación; su uso en el proceso provee un conjunto de técnicas de comunicación, computación y equipamiento de oficina utilizadas con la finalidad de aumentar la productividad y calidad de la gestión de la operación.

Un gran riesgo que se corre en la automatización, es el de adquirir productos sin haber establecido la estrategia de manejo de la información. Muchas veces se adquieren sistemas preestablecidos que no se adaptan completamente a la organización, y que terminan por formar grupos aislados o generar montones de información inutilizable; así mismo se confunden los conceptos y se cree que un mar de información será la solución; no sólo es cuestión de velocidad, sino de tener la información que realmente se requiere: información vital y oportuna

Además de los beneficios que aporta un sitio web a todos los usuarios interesados provenientes de una institución educativa que pueden ser primaria, secundaria, preparatoria e incluso universidades que desean tener los servicios de "Laboratorios de divulgación", cuyo concepto de transportar y difundir la ciencia y tecnología no tiene igual en el estado.

Teniendo así en Guanajuato 10,608 instituciones de educación básica, 1,217 de educación media superior y 232 superior disponibles para obtener los beneficios que otorga la difusión de ciencia y tecnología en cada uno de los participantes, estimulando las vocaciones científicas en la niñez y la juventud del estado, además de fortalecer la cultura científica de los ciudadanos y vincularlos a la comunidad científica y tecnológica, mediante actividades y experiencias educativas basadas en enfoques interactivos, experimentales $\mathrm{y}$ participativos.
En el presente artículo se explica la realización de un sitio web para la administración de solicitudes de visitas de divulgación científica en el Instituto Tecnológico superior de Irapuato.

\section{Estado del arte}

\section{Sistema de información}

Un sistema de información es un conjunto de elementos que interactúan entre sí, con el fin de apoyar las actividades de una empresa o negocio. Estos elementos son de naturaleza diversa en incluyen:

- $\quad$ El equipo computacional: es el hardware necesario para que el sistema de información opere. Lo constituyen y el equipo periférico

- $\quad$ El recurso humano que interactúa con el sistema de información: las personas que utilizan el sistema, lo alimentan con datos o utilizan los resultados que genere.

Los datos o información fuente: son todas las entradas que el sistema necesita para generar la información que se desea. Los programas que ejecuta la computadora y que producen diferentes tipos de resultados: los programas procesan los datos de entrada y generan los resultados que se esperan.

- $\quad$ Las telecomunicaciones: básicamente el hardware y el software que transmiten en forma electrónica texto, datos, imágenes y voz

- Procedimientos: que incluyen las políticas y reglas de operación, tanto en la parte funcional del proceso de negocio, como los mecanismos para hacer trabajar una aplicación en la computadora

Un sistema de información realiza cuatro actividades básicas, las cuales se definen a continuación.

- $\quad$ Entrada de información: Es el ingreso de los datos en el sistema de información. Las entradas pueden ser manuales, las proporciona el usuario o automáticas, datos o información que provienen o se toman de otros sistemas o módulos, lo que se denomina interfaces automáticas 
- Almacenamiento de información: es una de las capacidades más importantes de una computadora, ya que permite al sistema recordar la información guardada en la sesión anterior. Esta información se almacena en estructuras de información denominadas archivos, en su versión simple, y bases de datos, en su modalidad

Procesamiento de información: Es la capacidad del sistema de información para efectuar cálculos de acuerdo con una secuencia de operaciones preestablecidas. Estos cálculos trabajan con datos de recién ingreso o con datos ya almacenados.

Salida de información: es la capacidad de un sistema de información para convertir la información procesada o los datos de entrada en información para el exterior.

\section{Ciclo de vida en cascada}

Primer modelo de proceso de desarrollo de software se derivó de procesos de ingeniería de sistemas más generales. Este modelo se nombra así debido a la cascada de una fase a otra, sus principales actividades son:

1. Análisis y definición de requerimientos.

2. Diseño del sistema y del software.

3. Implementación y prueba de unidades.

4. Integración y prueba de unidades.

5. Funcionamiento y mantenimiento.

\section{Descripción del Método}

\section{Análisis y definición de requerimientos}

La primera fase de este ciclo consiste en conocer $\mathrm{y}$ detallar las características funcionales $\mathrm{y}$ principales que el sistema de información tendrá, para este proyecto se realizaron entrevistas, y de estas se obtuvieron los requerimientos funcionales y no funcionales así como las diferentes páginas que debería contener el sistema web. El siguiente diagrama se representa las ventanas del sistema y mediante flechas las navegaciones entre las mismas. Se ha de representar los caminos más significativos Fig.1

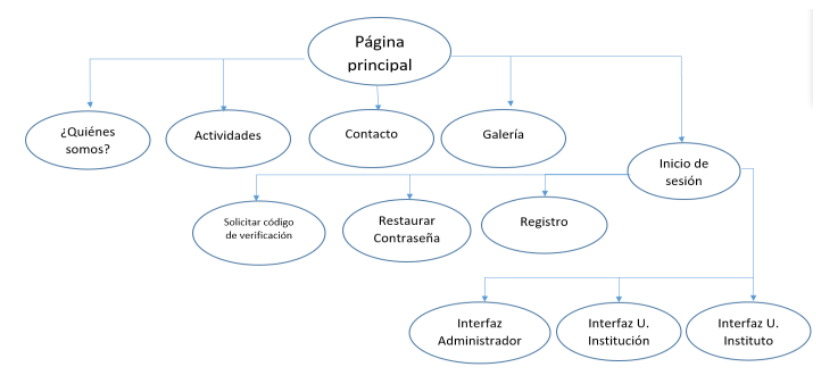

Figura 1 Diagrama Sistema Web

\section{Diseño del sistema y del software}

Durante esta etapa se construye un esquema conceptual representado por los objetos del dominio, las relaciones y colaboraciones existentes establecidas entre ellos. La idea principal es unificar una serie de tareas para obtener el diseño de navegación de la aplicación. En base a la obtención de requisitos funcionales del sistema se desarrollaron las siguientes interfaces. En la sección actividades de divulgación, las actividades son presentadas en "Cartas", las cuales contienen la información general de cada actividad. Fig.2.

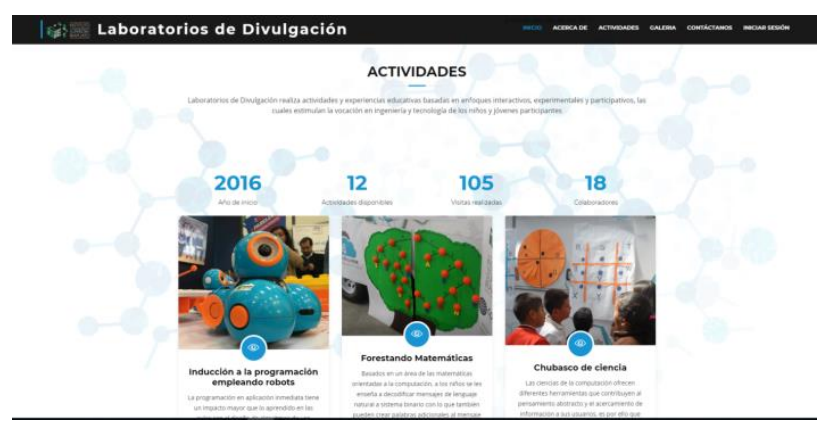

Figura 2 Actividades Disponibles

El registro del sistema de Laboratorios de Divulgación está destinado a aquellas instituciones educativas del estado de Guanajuato las cuales deseen obtener los servicios de Laboratorios de Divulgación. Dicho registro requiere ciertos datos divididos en Fig.3:

Datos del usuario: Datos de aquella persona proveniente de la Institución educativa puede o no ser el encargado de la institución, el cual hará uso del sistema

- Datos de la institución: Datos de la institución relacionada al usuario que realiza dicho registro. 


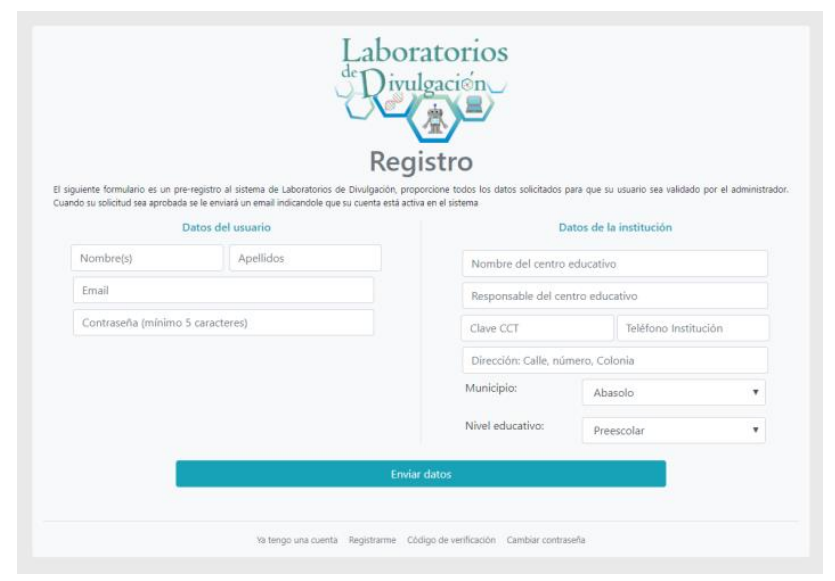

Figura 3 Registro

Cuando un usuario realice una solicitud de visitas lo cual se explicará más adelante, los administradores pueden visualizar dichas solicitudes, teniendo así la opción de aceptarlas o denegarlas Fig.4.

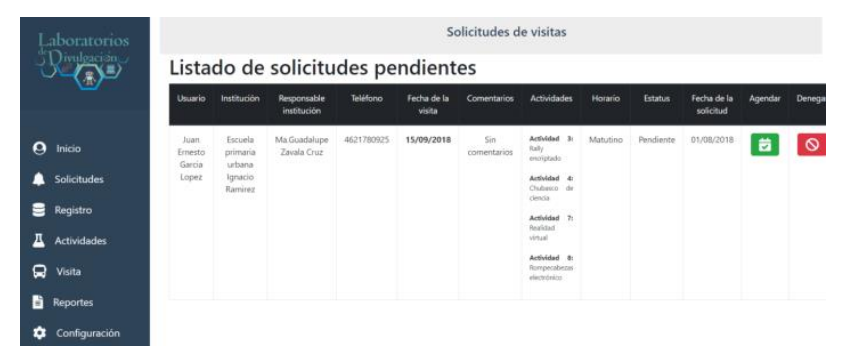

Figura 4 Gestion Solicitudes

De igual forma los administradores del sistema pueden agregar y eliminar actividades según con las que se cuenten para su impartición de los talleres de divulgación Fig.5.

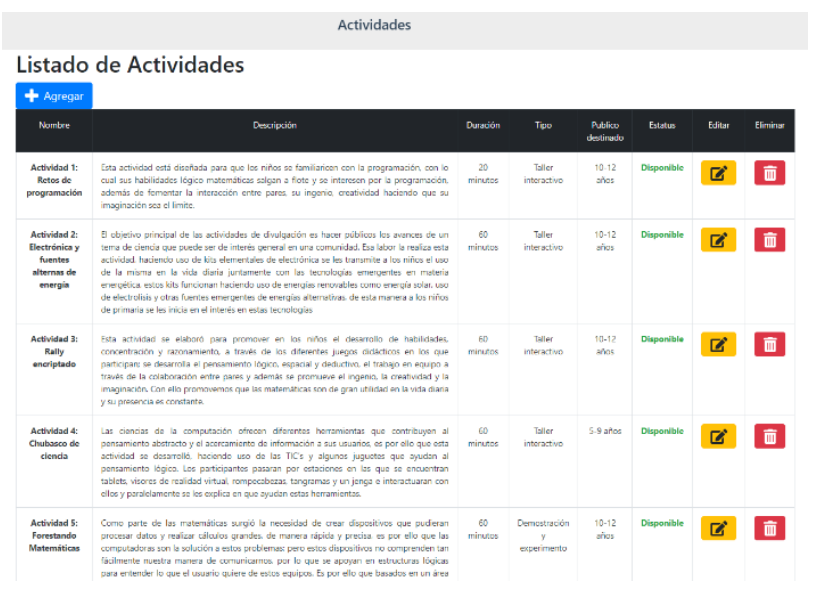

Figura 5 Edición de Actividades

\section{Implementación y prueba de unidades}

Después de la realización del sistema web, se precedió a realizar la instalación del servidor que lo ejecutara, dentro de las instalaciones del Instituto tecnológico superior de Irapuato.
El cual se probó durante un semestre agosto diciembre 2018 en el cual se pudieron realizar correcciones a bugs del sistema así como contemplar la funcionalidad del mismo y la integridad de la información. A continuación podemos ver el correo generado por el sistema que reciben los solicitantes así como una de las cartas de solicitud de servicios.Fig.6 y Fig.7.

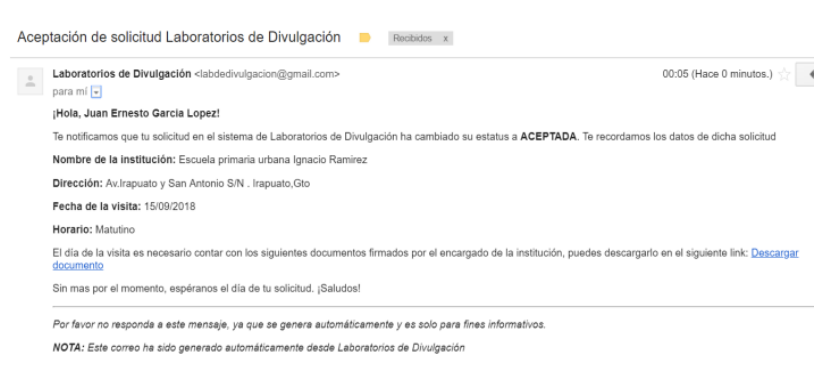

Figura 6 Correo Solicitante

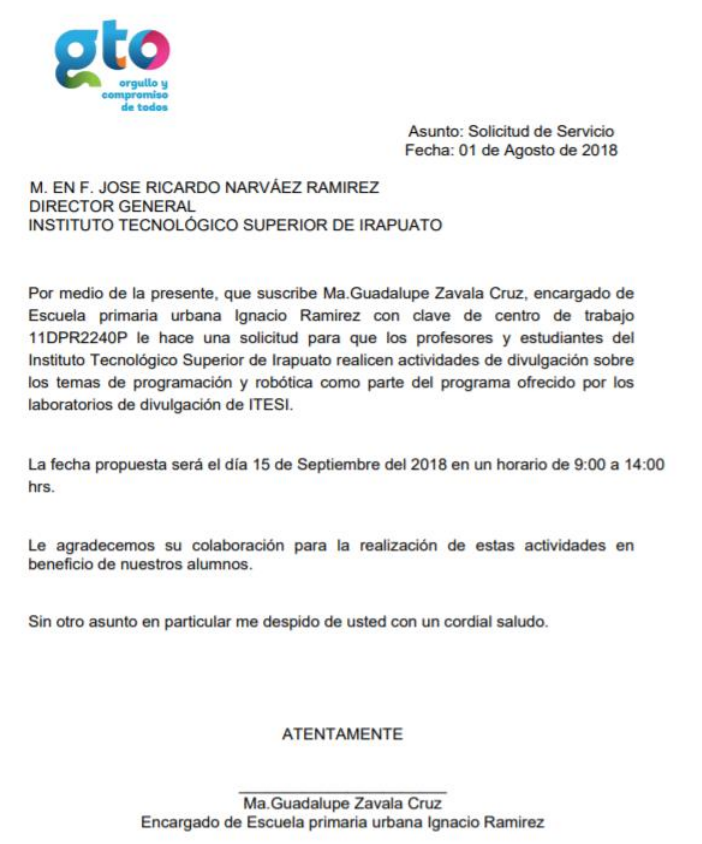

Figura 7 Solicitud de Servicio

\section{Resultados}

Sin el uso de sistema y su sitio web se tienen las siguientes tareas manuales como parte del procedimiento de solicitud de visita las cuales fueron descritas en la problemática del presente documento:

- Contactar a las personas provenientes de instituciones educativas para dar a conocer el proyecto y las actividades disponibles -15 minutos 
- Notificar al departamento de investigación y a los integrantes de Laboratorios de divulgación la solicitud de una visita - 45 minutos

- Generar documentos de solicitud de visita y agradecimiento, y enviarlos a los solicitantes -60 minutos

Consultar las visitas realizadas -30 minutos

Generar gráficos recopilando información del proyecto -30 minutos

Notificar a las personas que realizaron una solicitud que la misma ha sido aceptada o rechaza -15 minutos

Con la implementación del sistema, tenemos el siguiente nuevo número de tareas manuales a realizar con su tiempo invertido cada una:

- $\quad$ Mantener las actividades actualizadas - 5 minutos

- Verificar que los usuarios registrados provengan de una Institución educativa 10 minutos

Actualizar el estado de una solicitud -1 minuto

Como puede observarse el número de actividades manuales se reducen y cambian, esto se debe a la automatización de las mismas dentro de la programación del sistema. Ademes se tiene los resultados obtenidos de las encuestas establecidas para el proyecto. Estas encuestas realizadas fueron en base a las características del modelo estándar ISO 9126 el cual es un estándar internacional para la evaluación de la calidad del software cuyas características se pueden observar en la tabla de la figura8.

\begin{tabular}{|l|l|}
\hline Caracteristicas & Subcaracteristicas \\
\hline Funcionalidad & $\begin{array}{l}\text { Aplicabilidad, exactitud, seguridad } \\
\text { interoperatividad. }\end{array}$ \\
\hline Fiabilidad & $\begin{array}{l}\text { Madurez, tolerancia a fallos, capacidad de } \\
\text { recuperación. }\end{array}$ \\
\hline Usabilidad & $\begin{array}{l}\text { Capacidad para ser entendido, capacidad para } \\
\text { ser operado, capacidad para ser aprendido, } \\
\text { capacidad de atracción. }\end{array}$ \\
\hline Eficiencia & $\begin{array}{l}\text { Comportamiento temporal, utilización de } \\
\text { recursos. }\end{array}$ \\
\hline Mantenibilidad & $\begin{array}{l}\text { Capacidad para ser analizado, capacidad para } \\
\text { ser cambiado, estabilidad, capacidad para ser } \\
\text { probado. }\end{array}$ \\
\hline Portabilidad & $\begin{array}{l}\text { Adaptabilidad, facilidad de instalación, } \\
\text { coexistencia, capacidad para ser reemplazado, } \\
\text { cumplimiento de la portabilidad. }\end{array}$ \\
\hline
\end{tabular}

Figura 8 ISO 9126
En cuanto a las encuestas se realiza una tabla con el porcentaje obtenido de cada característica de calidad evaluada, obteniendo así el promedio de la evaluación de calidad del sistema.

\begin{tabular}{|l|c|}
\hline \multicolumn{2}{|c|}{ Resultado obtenido (\% Cumplimiento) } \\
\hline Encuesta 1 Usabilidad & $\mathrm{X}=13 / 15 * 100=86.66 \%$ \\
\hline Encuesta 2 Seguridad & $\mathrm{X}=3 / 4 * 100=75 \%$ \\
\hline $\begin{array}{l}\text { Encuesta } \\
\text { Mantenibilidad }\end{array}$ & $\mathrm{X}=6 / 6 * 100=100 \%$ \\
\hline Encuesta 4 Portabilidad & $\mathrm{X}=4 / 6 * 100=66 \%$ \\
\hline \% Total de cumplimiento & $81.9 \%$ \\
\hline
\end{tabular}

Como se puede observar en se tiene una disminución de tareas manuales por consiguiente un menor tiempo invertido para el proceso de solicitud de visita, lo cual lleva a una reducción de esfuerzo, un aumento en los beneficios en cuanto a la divulgación del proyecto, almacenamiento de la información y la mejora de procesos, se concluye que el sistema afecta positivamente a las actividades de Laboratorios de divulgación. Además, que este sistema tiene un cumplimiento de calidad del 81.9\% lo cual indica la satisfacción del cliente al obtener un producto bajo normas de calidad dentro de su ramo.

El sistema web también cuenta con reportes los cuales ayudaran a la toma de decisiones futuras que puedan beneficiar al ITESI, al crecimiento de laboratorio de divulgación y a toda la comunidad de estudiantes de los niveles básicos. Se muestra el reporte de actividades solicitadas por las instituciones Fig.9.

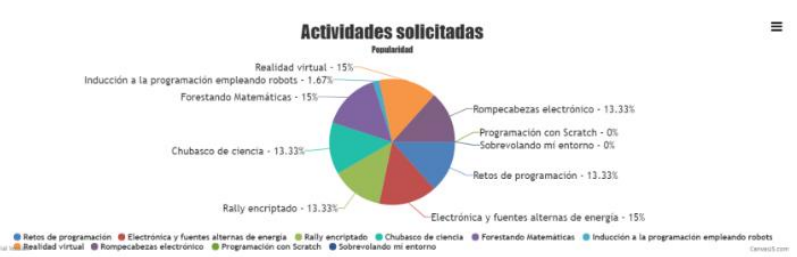

Figura 9 Reporte Actividades Solicitadas

De igual forma se puede tener acceso a información referente a las solicitudes por mes, para sí ver cuáles son los meses con mayor afluencia de estas.Fig.10. 


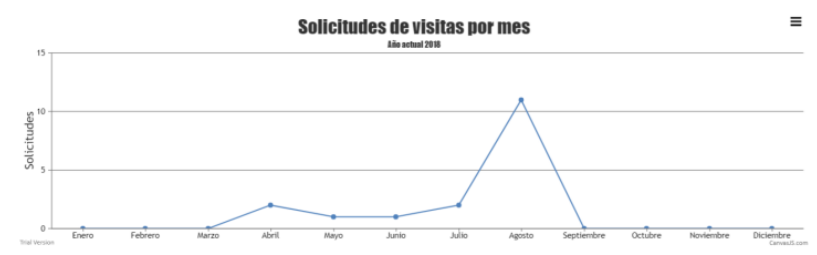

Figura 10 Reporte de Visitas Realizadas

\section{Agradecimiento}

Agradécenos al Instituto Tecnológico Superior de Irapuato, por las facilidades otorgadas para la realización de este proyecto así como al MTI. José Manuel Aguilera López encargado del laboratorio de divulgación, por la confianza brindada para la realización de este proyecto.

\section{Conclusiones}

Con la implementación del sistema se logró un incremento en las solicitudes recibidas en 42 solicitudes que representa un $30 \%$ y la atención a éstas con un incremento del $40 \%$ atendiendo un total de 17 comparada con el año anterior, esperando esto siga incrementado. Teniendo en cuenta una mejora continua del proyecto, se recomienda a desarrolladores interesados contribuir a la adición de más herramientas y servicios que este sistema pueda otorgar.

\section{Referencias}

Esposito, D. (2016). Modern Web Development. Microsoft Press .

Guillermo, P., \& Ludmila, R. (2015). Ingenieria de Software. Autonoma de Buenos Aires: Alfaomega Ediciones.

LÓPEZ ROMÁN, L. (2006). Metodología de la Programación Orientada a Objetos. México: Alfaomega.

usr.code. (16 de 05 de 2019). ingsw.pbworks.com. Obtenido de https://ingsw.pbworks.com/f/Ciclo+de+Vida+d el+Software.pdf

Vidal, C. L., Cabezas, C., Parra, J. H., \& López, L. P. (2015). Experiencias Prácticas con el Uso del Lenguaje de Programación Scratch para. Centro de Información Tecnológica, 23-32. 